\title{
Phylogenetic inferences of Atelinae (Platyrrhini) based on multi-directional chromosome painting in Brachyteles arachnoides, Ateles paniscus paniscus and Ateles b. marginatus
}

\author{
E.H.C. de Oliveira, ${ }^{a, b, c *}$ M. Neusser, ${ }^{a},{ }^{*}$ J.C. Pieczarka, ${ }^{b}$ C. Nagamachi, ${ }^{b}$ \\ I.J. Sbalqueiroc ${ }^{c}$ and S. Müllera \\ a Institut für Anthropologie und Humangenetik, Department Biologie II, Ludwig-Maximilians-Universität, Munich (Germany); \\ b Laboratório de Citogenética, Depto de Genética, Universidade Federal do Pará, Belém, PA (Brazil); \\ c Laboratório de Citogenética Animal, Depto de Genética, Universidade Federal do Paraná, Curitiba, PR (Brazil)
}

\begin{abstract}
We performed multi-directional chromosome painting in a comparative cytogenetic study of the three Atelinae species Brachyteles arachnoides, Ateles paniscus paniscus and Ateles belzebuth marginatus, in order to reconstruct phylogenetic relationships within this Platyrrhini subfamily. Comparative chromosome maps between these species were established by multi-color fluorescence in situ hybridization (FISH) employing human, Saguinus oedipus and Lagothrix lagothricha chromosome-specific probes. The three species included in this study and four previously analyzed species from all four Atelinae genera were subjected to a phylogenetic analysis on the
\end{abstract}

basis of a data matrix comprised of 82 discrete chromosome characters. The results confirmed that Atelinae represent a monophyletic clade with a putative ancestral karyotype of $2 n=$ 62 chromosomes. Phylogenetic analysis revealed an evolutionary branching sequence $\{$ Alouatta $\{$ Brachyteles $\{$ Lagothrix and Ateles $\}\}$ in Atelinae and \{Ateles belzebuth marginatus $\{$ Ateles paniscus paniscus \{Ateles belzebuth hybridus and Ateles geoffroyi $\}\}$ in genus Ateles. The chromosomal data support a reevaluation of the taxonomic status of Ateles $b$. hybridus.

Copyright $\odot 2005$ S. Karger AG, Basel
The subfamily Atelinae (Atelidae, Platyrrhini) comprises the four genera Brachyteles (woolly spider monkeys), Lagothrix (woolly monkeys), Ateles (spider monkeys) and Alouatta (howler monkeys) and is accepted as a monophyletic clade by most authors. The relationship of Brachyteles with the other genera of Atelinae (Atelidae, primates) is still controversial; however it is considered to be the sister group of Lagothrix in most of the recently proposed phylogenies based on molecular data (Schneider et al., 1993; Canavez et al., 1999; Meireles et al., 1999; von Dornun and Ruvolo, 1999), as well as G-banded chromosome analysis (Péquignot et al., 1985). DNA sequence

\footnotetext{
Received 27 October 2003; manuscript accepted 24 December 2003.

Request reprints from: Dr. Stefan Müller

Department Biologie II, Humangenetik, Ludwig-Maximilians-Universität

Richard-Wagner-Strasse 10, DE-80333 Munich (Germany)

telephone: +49-89-21806728; fax: +49-89-21806719

e-mail: S.Mueller@lrz.uni-muenchen.de
}

analysis grouped Brachyteles and Lagothrix closest to each other, followed by Ateles and finally Alouatta (Schneider et al., 1993, 1996; Harada et al., 1995; Porter et al., 1997a, b; Meireles et al., 1999). Morphological studies have led to a variety of different arrangements of the four Atelinae genera. Ford (1986) presented a trichotomy involving Ateles, Brachyteles and Lagothrix, with Alouatta as sister group to this clade, while Rosenberger et al. (1992) grouped Brachyteles and Ateles closest to each other, followed by Lagothrix and then Alouatta. Kay (1990) identified two sister subclades, one formed by Alouatta and Brachyteles and the second grouping Lagothrix and Ateles.

The taxonomy within genus Ateles is not yet fully resolved. According to Froehlich et al. (1991) the genus is comprised of three species and 8 subspecies, whereas Collins and Dubach (2000) listed four species (Table 1). The latter investigators per-

\footnotetext{
Both authors contributed equally to this work.
}

\begin{tabular}{lll}
\hline KARGER & $\begin{array}{l}\text { Fax +4161306 12 34 } \\
\begin{array}{l}\text { E-mail karger@karger.ch } \\
\text { www.karger.com }\end{array}\end{array}$ & 0301-0171/05/1083-0183\$22.00/0
\end{tabular}

Accessible online at: www. karger.com/cgr 
Table 1. Taxa of the genus Ateles recognized by Collins and Dubach (2000)

\begin{tabular}{|c|c|c|c|}
\hline Species & $2 n$ & G-banding/chr. nomenclature & Cross species FISH \\
\hline A. paniscus paniscus & 32 & Pieczarka et al (1989) & this study \\
\hline A. belzebuth belzebuth & 34 & Medeiros et al. (1997) & --- \\
\hline A. belzebuth marginatus & 34 & Medeiros et al. (1997) & this study \\
\hline A. belzebuth chamek ${ }^{\mathrm{a}}$ & 34 & Medeiros et al. (1997) & --- \\
\hline A. hybridus ${ }^{\mathrm{b}}$ & 34 & Medeiros et al. (1997) & Garcia et al. (2002) \\
\hline A. geoffroyi & 34 & Kunkel et al. (1980) & Morescalchi et al. (1997) \\
\hline $\begin{array}{ll}\text { a } & \text { Formerly classified a } \\
\text { b } & \text { Formerly classified a }\end{array}$ & ebut & $\begin{array}{l}\text { chamek (Kellogg and Goldman } \\
\text { hybridus (Froehlich et al., } 199\end{array}$ & \\
\hline
\end{tabular}

formed mitochondrial COII and D-loop sequence comparison and placed $A$. p. paniscus as the most basal, followed by $A$. $b$. hybridus with $A$. b. marginatus and $A$. geoffroyi as the last to diverge.

The genera Brachyteles and Lagothrix share the highest diploid chromosome number found among New World monkeys ( $2 \mathrm{n}=62$; Dutrillaux et al., 1980; Péquignot et al., 1985). Based on R-banding patterns of these genera, Péquignot et al. (1985) suggested that the karyotype of Brachyteles is more ancestral compared to that of Lagothrix. According to these investigators, the differences observed between their karyotypes are due to the occurrence of three pericentric inversions or centromeric shifts, a reciprocal translocation or insertion and the presence of additional heterochromatin segments in Lagothrix. Dutrillaux et al. (1980) placed Lagothrix as the sister group of Ateles in a study that included these two genera and Brachyteles, but no species of genus Alouatta. In contrast to Brachyteles and Lagothrix, Ateles has one of the lowest chromosome numbers among Platyrrhini monkeys, which ranges from 32 in $A$. p. paniscus to 34 in all the other analyzed Ateles species (Kunkel et al., 1980; de Boer and Bruijn, 1990; Medeiros et al., 1997) (Table 1). Based on banding patterns, Medeiros et al. (1997) proposed a phylogeny involving the different species of Ateles, placing the karyotype of $A$. p. chamek as the most basal, followed by $A$. p. paniscus, and $A$. geoffroyi as the most derived. In addition, the investigators made assumptions about the biogeography and radiation of the group, suggesting that speciation in Ateles was strongly influenced by the quaternary refuge formation. The results of the analysis of mitochondrial DNA sequence variation (Collins and Dubach, 2000), however, placed $A$. p. paniscus as the most basal species of this genus.

In recent years, some Atelinae species were analyzed with human chromosome painting probes. These included Lagothrix lagothricha (Stanyon et al., 2001), Ateles geoffroyi (Morescalchi et al., 1997), Ateles belzebuth hybridus (Garcia et al., 2002) and five Alouatta species (Consigliere et al., 1996, 1998; de Oliveira et al., 2002). The findings showed that the majority of associations of human homologous segments found in Neotropical primates so far are also observed in Atelinae: $3 \mathrm{a} / 21$, 5a/7a, 10a/16a, 8a/18, 2b/16b, 14/15a $\mathrm{a}_{1}$. In addition, Atelinae exclusively share derived fissions of chromosome $1,4,5$ and 15 homologs that resulted in chromosome forms $1 \mathrm{a}_{1}, 1 \mathrm{a}_{2}, 4 \mathrm{a}$, association of $4 \mathrm{~b} / 15 \mathrm{a}_{2}, 4 \mathrm{c}$, inversion $7 \mathrm{a} / 5 \mathrm{a} / 7 \mathrm{a}$ and $5 \mathrm{~b}$, indicating that this group is of monophyletic origin.

With the aim of reconstructing the phylogenetic relationship between the four Atelinae genera and to obtain a deeper insight into chromosome evolution in genus Ateles, we analyzed the karyotypes of $B$. arachnoides, Ateles p. paniscus and Ateles $b$. marginatus by multi-directional chromosome painting, employing human, Saguinus oedipus and L. lagothricha chromosome-specific probes. The integration of our results with previous publications on Atelinae comparative molecular cytogenetics allowed us to use chromosomal data in a phylogenetic analysis comprising all the genera of Atelinae, as well as to propose a chromosomal phylogeny for Atelinae including four Ateles species.

\section{Materials and methods}

\section{Cell samples}

Chromosome preparations for in situ hybridization experiments were obtained from whole-blood cultures of one individual of $B$. arachnoides (BAR), kept at Centro de Primatologia do Rio de Janeiro (Rio de Janeiro, Brazil), one male and one female L. lagothricha, one female A. b. marginatus, kept at Centro Nacional de Primatas (Ananindeua, PA, Brazil) and one individual of A. p. paniscus, kept at Parque Zoobotânico do Museu Emilio Goeldi (Belém, PA, Brazil). The diploid numbers found in all the specimens were as described before (Koiffmann, 1977; Dutrillaux et al., 1980; Péquignot et al., 1985; Medeiros et al., 1997).

\section{$G$-banding, probe composition, in situ hybridization and detection}

G-banding was performed according to Garcia et al. (2000). Human and New World monkey chromosome-specific painting probes were previously described in Stanyon et al. (2001) and Müller et al. (2001), respectively. Multi-color probe sets H1-H4 comprised all 24 human and S1-S4 all 24 S. oedipus chromosome-specific probes. Probe set L1 was composed of those $L$. lagothricha chromosome paint probes that provided more detailed subchromosomal information than those derived from $S$. oedipus homologs (Neusser et al., 2001, de Oliveira et al., 2002). All probe sets were labeled by DOPPCR in Boolean combinations in the presence of Biotin-dUTP, DigoxigenindUTP (Roche) and TAMRA-dUTP (Applied Biosystems/PE). Hybridization in situ and detection were carried out exactly as described in Neusser et al. (2001) and de Oliveira et al. (2002).

\section{Microscopy and image analysis}

Metaphases were analyzed with a cooled CCD camera (Photometrics C250/A equipped with a KAF1400 chip) coupled to a Zeiss Axiophot microscope. Merging of chromosome images was performed using the software Adobe Photoshop version 3.0. Chromosomes were identified by computerenhanced DAPI banding (SmartCapture VP 1.4, Digital Scientific, Cambridge, UK).

Maximum parsimony analysis (PAUP)

Table 1 summarizes the Ateles species included in this phylogenetic analysis. A data matrix was established (Table 2 and 3), based on the presence or absence of discrete chromosomal homology characters in the three species $B$. arachnoides, $A$. p. paniscus and $A$. b. marginatus, included in our study, in four previously published Atelinae species and in the phylogenetic outgroup Cebus apella. These characters (1) comprised 76 conserved syntenic seg- 
Table 2. Summary of characters (homologous chromosomes and syntenic association of homologous chromosome segments) that were used to assemble the binary data matrix $(\mathrm{i}=$ inversion, $\mathrm{f}=$ fission)

\begin{tabular}{|l|l|l|l|l|l|}
\hline $1.1 \mathrm{a}_{1}$ & \multicolumn{1}{|c|}{$2.1 \mathrm{a}_{1} / 1 \mathrm{a}_{2}$} & $3.1 \mathrm{a}_{1} / 1 \mathrm{c}$ & $4.1 \mathrm{a}_{1} / 4 \mathrm{~b}$ & $5.1 \mathrm{a}_{1} / 6$ & $6.1 \mathrm{a}_{1} / 14$ \\
\hline $7.1 \mathrm{a}_{2}$ & $8.1 \mathrm{a}_{2} / 5 \mathrm{~b}$ & $9.1 \mathrm{a}_{2} / 7 \mathrm{~b}$ & $10.1 \mathrm{~b}$ & $11.1 \mathrm{~b} / 2 \mathrm{~b}$ & $12.1 \mathrm{c}$ \\
\hline $13.1 \mathrm{c} / 1 \mathrm{~b}$ & $14.1 \mathrm{c} / 6$ & $15.1 \mathrm{c} / 6 \mathrm{i}$ & $16.2 \mathrm{a}$ & $17.2 \mathrm{a} / 2 \mathrm{~b}$ & $18.2 \mathrm{a} / 3 \mathrm{~b}$ \\
\hline $19.2 \mathrm{a} / 10 \mathrm{~b}$ & $20.2 \mathrm{a} / 20$ & $21.2 \mathrm{~b}$ & $22 .(2 \mathrm{~b} / 16 \mathrm{~b})_{1}$ & $23 .(2 \mathrm{~b} / 16 \mathrm{~b}) \mathrm{f}$ & $24 .(2 \mathrm{~b} / 16 \mathrm{~b})_{2}$ \\
\hline $25.3 \mathrm{a} / 3 \mathrm{~b}$ & $26.3 \mathrm{a} / 6$ & $27.3 \mathrm{a} / 21$ & $28.3 \mathrm{~b}$ & $29.3 \mathrm{~b} / 3 \mathrm{c}$ & $30 .(3 \mathrm{~b} / 15 \mathrm{~b})$ \\
\hline $31.3 \mathrm{c}$ & $32.3 \mathrm{c} / 7 \mathrm{~b}$ & $33.3 \mathrm{c} / 13 \mathrm{~b}$ & $34.3 \mathrm{c} / 15 \mathrm{~b}$ & $35.4 \mathrm{a}$ & $36.4 \mathrm{a} / 4 \mathrm{~b}$ \\
\hline $37.4 \mathrm{~b}$ & $38.4 \mathrm{~b} / 4 \mathrm{c}$ & $39.4 \mathrm{~b} / 15 \mathrm{a}_{2}$ & $40.4 \mathrm{c}$ & $41.4 \mathrm{c} / 7 \mathrm{a}$ & $42.4 \mathrm{c} \mathbf{i}$ \\
\hline $43.4 \mathrm{c} / 16 \mathrm{~b}$ & $44.5 \mathrm{a} / 5 \mathrm{~b}$ & $45.5 \mathrm{a} / 7 \mathrm{a}$ & $46 .(5 \mathrm{a} / 7 \mathrm{a}) \mathrm{i}$ & $47.5 \mathrm{~b} \mathbf{i}$ & $48.5 \mathrm{~b}$ \\
\hline $49.5 \mathrm{~b} / 7 \mathrm{a}$ & $50.5 \mathrm{~b} / 8 \mathrm{~b}$ & $51.5 \mathrm{~b} / 12$ & $52.7 \mathrm{a} / 7 \mathrm{~b}$ & $53.7 \mathrm{~b}$ & $54.8 \mathrm{a} / 8 \mathrm{~b}$ \\
\hline $55.8 \mathrm{a} / 16 \mathrm{a}$ & $56.8 \mathrm{a} / 18$ & $57.8 \mathrm{~b} \mathbf{i}$ & $58.8 \mathrm{~b}$ & $59.8 \mathrm{~b} / 12$ & $60.9 / 18$ \\
\hline $61.9 / 22$ & $62.10 \mathrm{a} / 10 \mathrm{~b}$ & $63 .(10 \mathrm{a} / 16 \mathrm{a})_{1}$ & $64 .(10 \mathrm{a} / 16 \mathrm{a}) \mathrm{i}$ & $65.10 \mathrm{~b}$ & $66.12 \mathbf{i}$ \\
\hline $67.12 / 15 \mathrm{a}_{1}$ & $68.13 \mathbf{i}$ & 69.14 & $70.14 / 15 \mathrm{a}_{1}$ & $71.15 \mathrm{a}_{1} / 15 \mathrm{a}_{2}$ & $72.15 \mathrm{a}_{1} / 15 \mathrm{~b}$ \\
\hline $73.15 \mathrm{a}_{2} / 15 \mathrm{~b}$ & $74.15 \mathrm{~b}$ & $75.15 \mathrm{~b} / 22$ & $76.16 \mathrm{a} / 16 \mathrm{~b}$ & $77.16 \mathrm{~b}$ & 78.18 \\
\hline $79.19 / 20$ & $80.20 \mathbf{i}$ & 81.21 & $82 . \mathrm{Y} / 15 \mathrm{~b}$ & & \\
\hline
\end{tabular}

Bold characters were derived by comparative G-banding analysis. The nomenclature of chromosome segments is following Neusser et al. (2001) and de Oliveira et al. (2002).

ments detectable by chromosome painting and associations thereof and (2) six characters obtained by G-banding pattern comparison (Table 2). The character nomenclature for human homologous segments detectable with human, $S$. oedipus and L. lagothricha probes paint probes followed Neusser et al. (2001) and de Oliveira et al. (2002): $1 \mathrm{a}_{1}(1 \mathrm{p} 21 \rightarrow$ pter $), 1 \mathrm{a}_{2}(1 \mathrm{p} 12 \rightarrow$ p21), 1c (q21 $\rightarrow$ q31), 1b (q32 $\rightarrow$ qter), 2a (q12 $\rightarrow$ qter), $2 b($ pter $\rightarrow$ q12), 3b $(\mathrm{p} 24 \rightarrow$ pter, p12 $\rightarrow$ p21, q12 $\rightarrow$ q13, q27 $\rightarrow$ qter), $3 \mathrm{c}(\mathrm{p} 21 \rightarrow \mathrm{p} 24, \mathrm{q} 13 \rightarrow \mathrm{q} 26)$, $3 \mathrm{a}(\mathrm{p} 12), 4 \mathrm{a} \quad(4 \mathrm{q} 31.3 \rightarrow \mathrm{qter}), 4 \mathrm{~b} \quad(4 \mathrm{q} 23 \rightarrow \mathrm{q} 31.2), 4 \mathrm{c}(4 \mathrm{pter} \rightarrow \mathrm{q} 22), 5 \mathrm{a}$ $(5 \mathrm{q} 31.3 \rightarrow$ qter $), 5 \mathrm{~b}(5 \mathrm{pter} \rightarrow \mathrm{q} 31.2), 6,7 \mathrm{a}(\mathrm{p} 22, \mathrm{q} 11, \mathrm{q} 21), 7 \mathrm{~b}(\mathrm{p} 11 \rightarrow \mathrm{p} 21$, $\mathrm{q} 11 \rightarrow \mathrm{q} 21, \mathrm{q} 22 \rightarrow \mathrm{qter}), 8 \mathrm{a}(8 \mathrm{p}), 8 \mathrm{~b}(8 \mathrm{q}), 9,10 \mathrm{a}(10 \mathrm{q}), 10 \mathrm{~b}(10 \mathrm{p}), 11,12,13 \mathrm{a}$ $\left(\mathrm{q} 14 \rightarrow\right.$ qter), $13 \mathrm{~b}(\mathrm{q} 11 \rightarrow \mathrm{q} 13), 14,15 \mathrm{a}_{1}(\mathrm{q} 11 \rightarrow \mathrm{q} 24), 15 \mathrm{a}_{2}(15 \mathrm{q} 13 \rightarrow \mathrm{q} 21.2)$ $15 \mathrm{~b}(\mathrm{q} 11 \rightarrow 13, \mathrm{q} 25 \rightarrow$ qter $), 16 \mathrm{a}(16 \mathrm{p}), 16 \mathrm{~b}(16 \mathrm{q}), 17,18,19,20,21,22, \mathrm{X}$ and $\mathrm{Y}$.

The data was subjected to maximum parsimony analysis (PAUP 4.0 software; Phylogenetic Analysis Using Parsimony, Swofford 1998), using the exhaustive search option. All characters had the same weight, based on the premise that chromosome rearrangements occur by equal chance. The relative stability of nodes was assessed by bootstrap estimates based on 1000 iterations. Each bootstrap replicate involved a heuristic parsimony search with 10 random taxon additions and tree-bisection-reconnection branch swapping. Two different PAUP analyses were performed: one of them including only characters based on FISH data, while the second included characters derived both by FISH and G-banding comparison.

\section{Results}

All human, S. oedipus and L. lagothricha sets of chromosome-specific probes delivered reproducible results when hybridized to metaphases of $B$. arachnoides (BAR), A. p. paniscus (APP) and $A$. b. marginatus (ABM), except for the human Ychromosome probe. Centromeric and heterochromatic chromosome regions were not hybridized by any of the paint probes, except for L. lagothricha chromosome-specific probes in $B$. arachnoides, where centromeric regions were weakly hybridized. Chromosome identification was performed on the basis of DAPI banding. In each experiment a minimum of 10 cells were examined. Figure $1 \mathrm{~A}-\mathrm{F}$ illustrates representative FISH experiments with these probe sets.

\section{Ateles belzebuth marginatus}

The diploid number of $A$. b. marginatus was 34, with a submetacentric $X$ chromosome. Figure $2 \mathrm{~A}$ illustrates the $\mathrm{G}$ banding patterns, following the chromosome nomenclature proposed by Medeiros et al. (1997), together with the homology of human and S. oedipus chromosomes. S. oedipus chromosome specific probes produced 44 signals: Only chromosome 11 was entirely conserved between $S$. oedipus and $A$. b. marginatus. All other chromosomes were rearranged and showed associations of $S$. oedipus homologs 1/3/15, 12/11/16/7/11, 1/9/17/9, 19/13/ $16,6 / 18,10 / 20,16 / 8 / 21,5 / 2,7 / 6,4 / 8,2 / 1$ and $22 / 9$.

Human painting probes produced 52 signals, with only three conserved syntenies on separate chromosomes: HSA 11, 13, 17 and X. Human chromosome 9 also remained conserved but was involved in a Robertsonian fusion. Association of human homologous segments 9/18/8a/16a/10a/16a, 12/15a 1 $14 / 1 \mathrm{a}_{1} / 4 \mathrm{~b} / 15 \mathrm{a}_{2}, 22 / 15 \mathrm{~b} / 3 \mathrm{~b} / 2 \mathrm{a}, 3 \mathrm{c} / 7 \mathrm{~b} / 1 \mathrm{a}_{2}, 5 \mathrm{~b} / 8 \mathrm{~b}, 16 \mathrm{~b} / 2 \mathrm{~b} / 1 \mathrm{~b}$, $1 a_{1} / 6 / 1 \mathrm{c}, 19 / 20,4 c / 7 \mathrm{a} / 5 \mathrm{a} / 7 \mathrm{a}, 21 / 3 \mathrm{a} / 6$ and $10 \mathrm{~b} / 2 \mathrm{a}$ were observed.

\section{Ateles paniscus paniscus}

Hybridization of human, S. oedipus and L. lagothricha chromosome-specific probes to $A$. p paniscus chromosomes $(2 \mathrm{n}=$ 32 , the chromosome nomenclature follows Pieczarka et al., 1989) revealed identical hybridization patterns for most chromosomes. Compared to A. b. marginatus. A. p. paniscus chromosome 4 showed association of human homologs $13 \mathrm{a} / 13 \mathrm{~b} / 3 \mathrm{c} / 7 \mathrm{~b} /$ $1 \mathrm{a}_{2}$ and can be derived from $A$. $b$. marginatus chromosomes 4 and 12 by a tandem fusion (Fig. 2B). A. p. paniscus chromosome 7 (association $1 \mathrm{a}_{1} / 6 / 1 \mathrm{c} / 6 / 1 \mathrm{c}$ ) differs from $A$. b. marginatus chromosome 7 by a pericentric inversion (Fig. 2C). A detailed comparative G-banding analysis between both Ateles species revealed no further intra-chromosomal rearrangements.

\section{Brachyteles arachnoides}

The G-banded karyotype of B. arachnoides with the homology maps between this species, human, $S$. oedipus and L. lagothricha chromosomes is shown in Fig. 2D. Chromosomes were ordered according to the nomenclature proposed by Péquignot et al. (1985). Human painting probes revealed 41 conserved homologous segments. S. oedipus probes produced 34 signals: Sixteen autosomal syntenic groups (5, 7-13, 15-19, 21 and 22), as well as the $\mathrm{X}$ and $\mathrm{Y}$ chromosome are conserved in $B$. arachnoides. The remaining chromosomes were homologous to more than one chromosome of Brachyteles. The S. oedipus Y chromosome specific probe weakly hybridized to the Y chromo- 

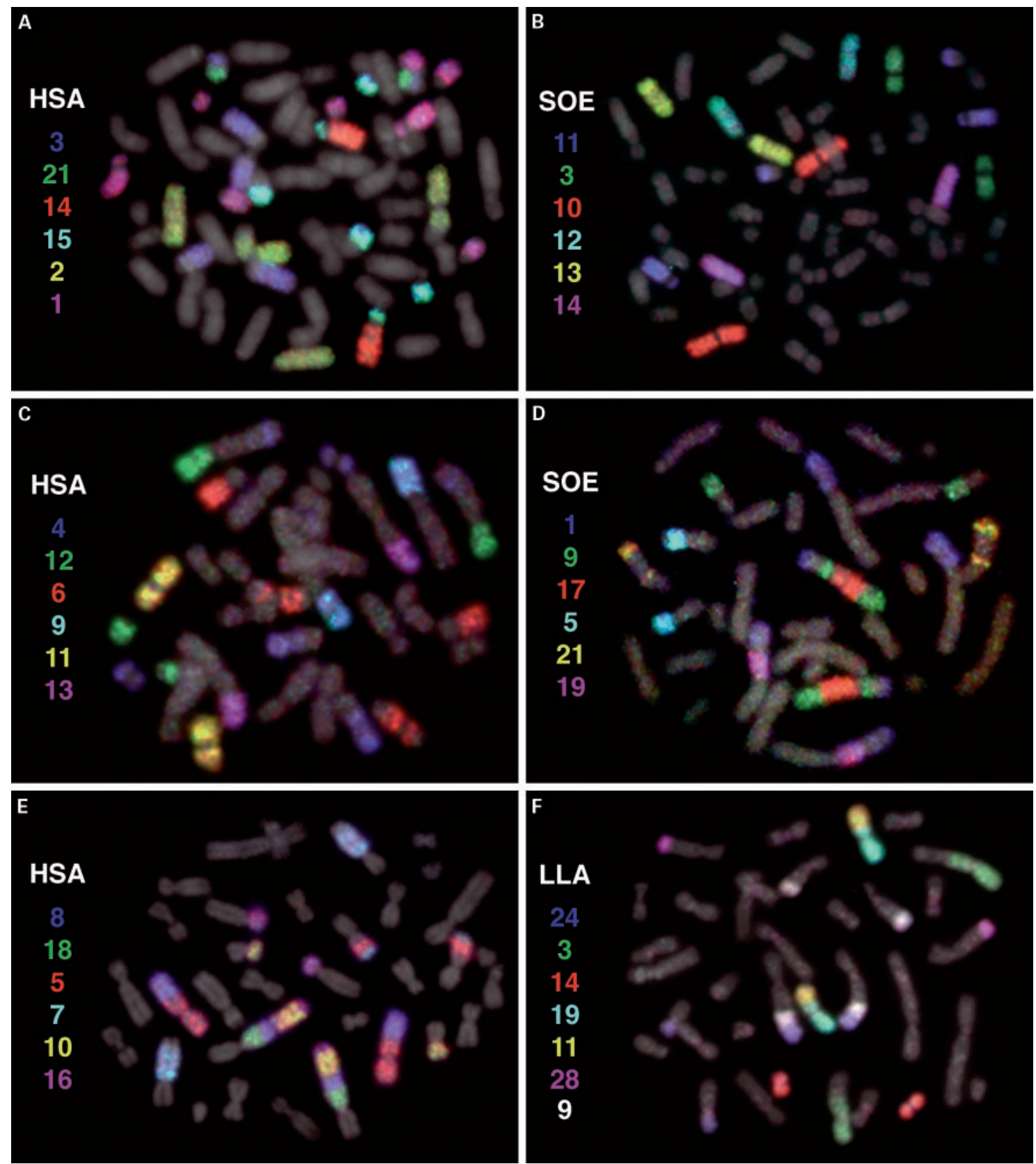

Fig. 1. Representative multi-color FISH experiments with human (HSA), S. oedipus (SOE) and L. lagothricha (LLA) painting probe sets to (A) and (B) B. arachnoides, (C) and (D) A. p. paniscus, (E) and (F) A. belzebuth marginatus metaphases. To visualize the hybridization pattern and chromosomal counter stain simultaneously, an overlay of both images is shown. Beside each metaphase the respective probe composition and false color assignment is given.

some of Brachyteles, confirming that this chromosome is the smallest of the complement.

Human homologous syntenic groups corresponding to chromosome pairs $6,9,11,12,13,17,19,20,22$ were conserved as separate chromosomes in $B$. arachnoides. The associations of human 2b/16b, 10a/16a, 8a/18, 5/7a, 14/15a and 3a/21 homologs frequently observed in New World monkeys were present. Furthermore, as observed in L. lagothricha and genus Alouatta, human chromosomes 1 and 4 are fragmented in four and three different chromosome segments, respectively, the chromosome $4 \mathrm{~b}$ homolog being translocated to chromosome 15a homologous material. The hybridization results were identical to those previously obtained in L. lagothricha (Stanyon et al., 2001), with the exception of an inversion leading to a chromosome form homologous to human chromosomes 10/16/10/16. Reinvestigation of $L$. lagothricha with the respective human probes, however, revealed the presence of this inversion also in the $L$. lagothricha individual studied (data not shown). 

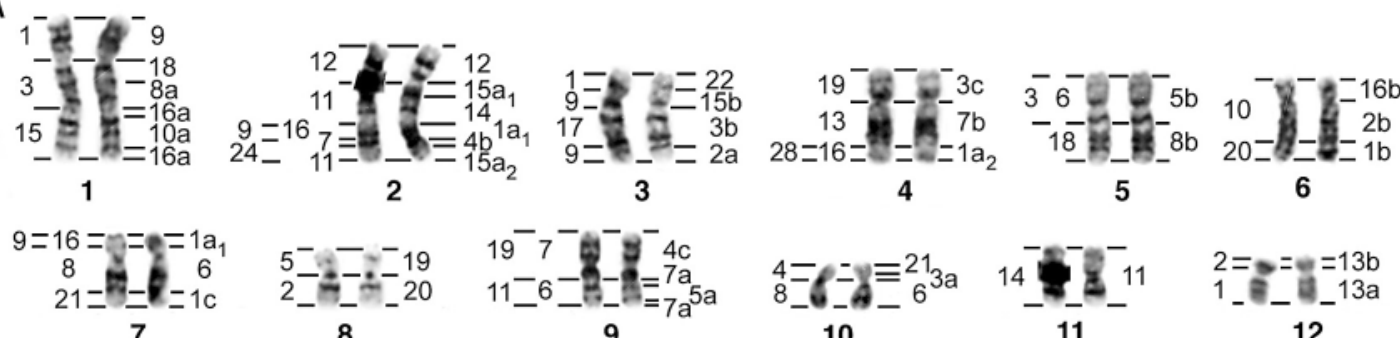

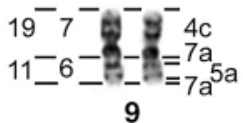
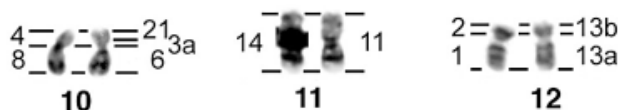

12
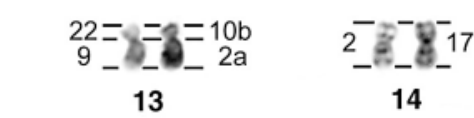

$144_{-}^{-}-8_{-}^{-}-4 a$

12- - -

16

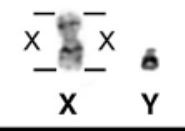

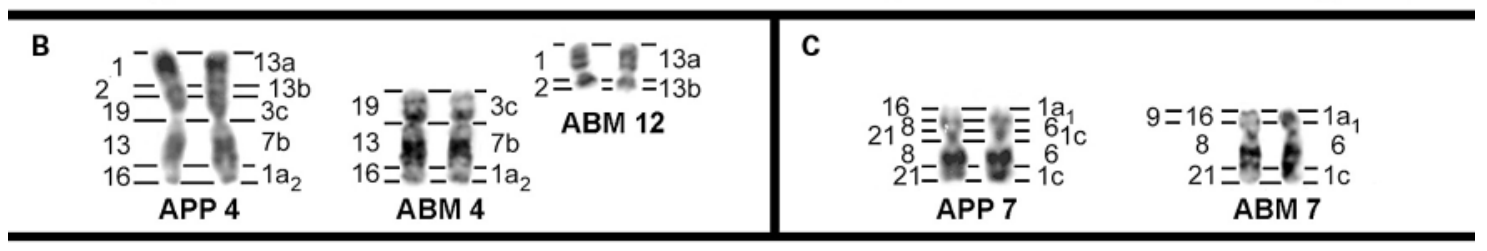

D
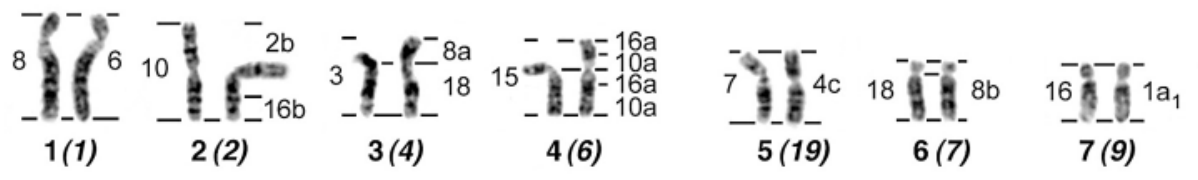

$-\mathrm{c}=8=7 \mathrm{a}$
$-8 \mathrm{a}$
$-7 \mathrm{a}$

$\begin{array}{cc}11-2=15 \mathrm{a}_{1} & 19-0_{-}^{-}-17 \\ -9(21) & \mathbf{1 0 ( 1 0 )}\end{array}$

$\frac{5-3-319}{11(13)}$

$7-8-4 a$

6 b-

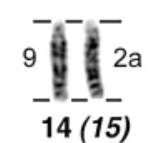

13 -
$-15(16)$

8 (11)

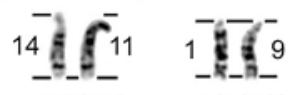 \\ 18 (18)}

$17-3 b$
$19(20)$

12 (14)

13 (3)

14 (15)
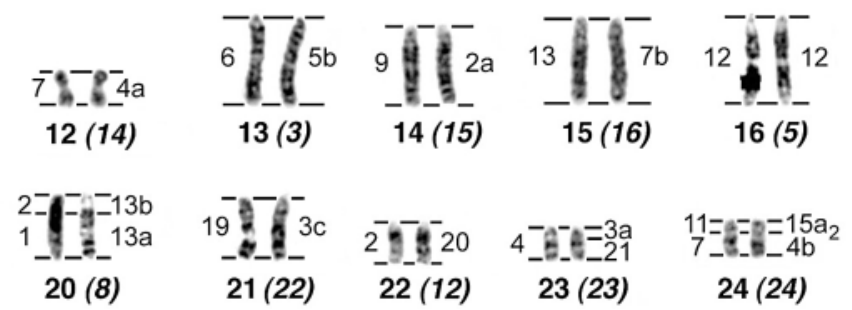

24 (24)
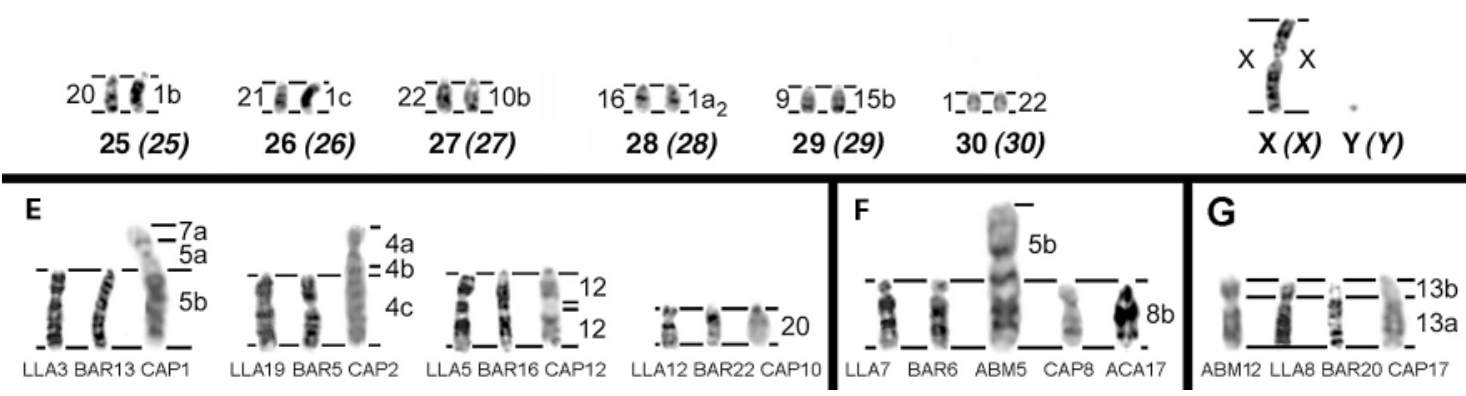

Fig. 2. (A) G-banded karyotype of $A$. belzebuth marginatus together with the assignment of human, $S$. oedipus and L. lagothricha chromosome-specific painting probes. Chromosome numbering (below each chromosome) follows citations given in Table 1 . Numbers beside chromosomes indicate human (right), S. oedipus (left) and L. lagothricha (far left) homologous chromosome regions, horizontal bars the borders of homologous regions. (B) and (C) Chromosomal differences between $A$. belzebuth marginatus (ABM) and A. p. paniscus (APP) detected by chromosome painting: (B) a tandem fusion and (C) a pericentric inversion. (D) G-banded karyotype of B. arachnoides,

\section{Comparative G-banding analysis}

Comparative G-banding analysis (Fig. 2E-G) revealed five intra-chromosomal rearrangements between $\mathrm{B}$. arachnoides and L. lagothricha not detectable by chromosome painting. A comparison with chromosome forms found in C. apella, Alouatta caraya and Ateles b. marginatus that were used as out- together with the assignment of human (right) and S. oedipus (left) homologous chromosome regions. Below each chromosome the numbering proposed by Pequignot et al. (1985) and in brackets the chromosome homology with $L$. lagothricha, following the nomenclature used by Stanyon et al. (2001) is given. (E-G) Chromosomal differences between $B$. arachnoides (BAR), L. lagothricha (LLA), A. belzebuth marginatus (ABM), Alouatta caraya (ACA) and Cebus apella (CAP) detected by G-banding, which were informative for the reconstruction of chromosomal phylogenies (see text for details).

group species to determine ancestral states, was performed. The results indicated that $B$. arachnoides chromosomes 13, 16 and 22 conserved Atelinae ancestral chromosome forms, from which $L$. lagothricha homologs may be derived by pericentric inversions, whereas $B$. arachnoides chromosome 5 showed a derived inversion (Fig. 2E). Furthermore, B. arachnoides chro- 
Table 3. Binary character matrix used in the parsimony analysis, comprising 82 characters $(0=$ absent, $1=$ present $)$

\begin{tabular}{|c|c|c|c|c|c|c|c|c|c|c|c|c|c|c|c|c|c|c|c|c|c|c|c|c|c|c|c|c|c|c|c|c|c|c|c|c|c|c|c|c|c|}
\hline char & 01 & 02 & 03 & 04 & 05 & 06 & 07 & 08 & 09 & 10 & 11 & 12 & 13 & 14 & 15 & 16 & 17 & 18 & 19 & 20 & 21 & 22 & 23 & 24 & 25 & 26 & 27 & 28 & 29 & 30 & 31 & 32 & 33 & 34 & 35 & $36 \mathrm{c}$ & 37 & 38 & 39 & 40 & 41 \\
\hline $\mathrm{AP}$ & 1 & 1 & 0 & 0 & 0 & 0 & 1 & 0 & 0 & 1 & 0 & 1 & 0 & 0 & 0 & 1 & 0 & 0 & 0 & 0 & 1 & 1 & 0 & 0 & 0 & 0 & 1 & & 0 & 0 & 1 & 0 & 0 & 0 & 1 & 1 & 1 & 1 & 0 & 1 & 0 \\
\hline AR & 1 & 0 & 0 & 0 & 0 & 0 & 1 & 0 & 0 & 1 & 0 & 1 & & c & 0 & 1 & ( & 0 & 0 & 0 & 1 & 1 & 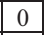 & 0 & 0 & 0 & & 1 & 0 & 0 & & 0 & 0 & 0 & 1 & 0 & 1 & 0 & 1 & 1 & 0 \\
\hline $\mathrm{LA}$ & 1 & 0 & 0 & 0 & 0 & 0 & 1 & \begin{tabular}{|l|} 
\\
\end{tabular} & 0 & 1 & 0 & 1 & 0 & 0 & 0 & 1 & 0 & 0 & 0 & 0 & 1 & 1 & 0 & 0 & 0 & 0 & 1 & 1 & 0 & 0 & 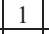 & 0 & 0 & 0 & 1 & 0 & 1 & 0 & 1 & 1 & 0 \\
\hline $\mathrm{GE}$ & 1 & 0 & 0 & 1 & 1 & 1 & 1 & \begin{tabular}{|l|}
0 \\
\end{tabular} & 1 & 1 & 1 & 1 & 0 & 1 & 1 & 1 & 0 & 1 & 1 & 0 & 1 & 1 & 0 & 1 & 0 & 1 & 1 & 1 & 0 & 1 & 1 & 1 & 0 & 0 & 1 & 0 & 1 & 0 & 1 & 1 & 1 \\
\hline PP & $=$ & 0 & 0 & 1 & 1 & 1 & 1 & 0 & 1 & 4 & 1 & 1 & 0 & 1 & 1 & 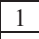 & 0 & 1 & 1 & 0 & 1 & 1 & 0 & 0 & 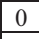 & 1 & 1 & 1 & 0 & 1 & 1 & 1 & 1 & 0 & 1 & 0 & 1 & 0 & 1 & 1 & 1 \\
\hline $\mathrm{BM}$ & 1 & 0 & 0 & 1 & 1 & 1 & 1 & \begin{tabular}{|l|}
0 \\
\end{tabular} & 1 & 1 & 1 & 1 & 0 & 1 & 0 & 1 & 0 & 1 & 1 & 0 & 1 & 1 & 0 & 0 & 0 & 1 & 1 & 1 & 0 & 1 & 1 & 1 & 0 & 0 & 1 & 0 & 1 & 0 & 1 & 1 & 1 \\
\hline $\mathrm{BH}$ & 1 & 0 & 0 & 1 & 1 & 1 & 1 & \begin{tabular}{|l|}
0 \\
\end{tabular} & 1 & 1 & 1 & 1 & 0 & 1 & 1 & 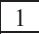 & 0 & 1 & 1 & 0 & 1 & 1 & 0 & 1 & 0 & 1 & 1 & 1 & 0 & 1 & 1 & 1 & 0 & \begin{tabular}{|l|} 
\\
\end{tabular} & 1 & 0 & 1 & 0 & 1 & 1 & 1 \\
\hline $\mathrm{HSA}$ & 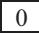 & 1 & 1 & 0 & 0 & 0 & 0 & 0 & 0 & 0 & 0 & 0 & 1 & 0 & 0 & 0 & 1 & 0 & 0 & 0 & 0 & 0 & 0 & 0 & 1 & 0 & 0 & 0 & 1 & 0 & 0 & 0 & 0 & 0 & 0 & 1 & 0 & 1 & 0 & 0 & 0 \\
\hline
\end{tabular}

\begin{tabular}{|c|c|c|c|c|c|c|c|c|c|c|c|c|c|c|c|c|c|c|c|c|c|c|c|c|c|c|c|c|c|c|c|c|c|c|c|c|c|c|c|c|c|}
\hline char & 42 & 43 & 44 & 45 & 46 & 47 & 48 & 49 & 50 & 51 & 52 & 53 & 54 & 55 & 56 & 57 & 58 & 59 & 60 & 61 & 62 & 63 & 64 & 65 & 66 & 67 & 68 & 69 & 70 & 71 & 72 & 73 & 74 & 75 & 76 & 77 & 78 & & 30 & 81 & 82 \\
\hline $\mathrm{AP}$ & 0 & 0 & 1 & 1 & 0 & 0 & 1 & 0 & 0 & 0 & 0 & 1 & 0 & 0 & 1 & 0 & 1 & 0 & 0 & 0 & 0 & 1 & 0 & 1 & 0 & 0 & 0 & 0 & 1 & 1 & 0 & \begin{tabular}{|l|} 
\\
\end{tabular} & 1 & 0 & 0 & 1 & 0 & 0 & O & 0 & 0 \\
\hline $\mathrm{AR}$ & 1 & 0 & 0 & 1 & 1 & (0 & 1 & 0 & 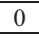 & 0 & 0 & 1 & 0 & 0 & 1 & 1 & 1 & 0 & 0 & 0 & 0 & 1 & 1 & 1 & 0 & 0 & 0 & 0 & 1 & 0 & 0 & 0 & 1 & 0 & 0 & 1 & 0 & 0 & 0 & 0 & 0 \\
\hline $\mathrm{LA}$ & 0 & 0 & 0 & 1 & 1 & 1 & 1 & 0 & 0 & 0 & 0 & 1 & 0 & 0 & 1 & 1 & 1 & 0 & 0 & 0 & 0 & 1 & 1 & 1 & 1 & 0 & 1 & 0 & 1 & 0 & 0 & 0 & 1 & 0 & 0 & 1 & 0 & 0 & 1 & 0 & 0 \\
\hline AGE & 0 & 0 & 0 & 1 & 1 & 0 & 1 & 0 & 1 & 1 & 0 & 1 & 0 & 1 & 1 & 1 & 1 & 1 & 1 & 0 & 0 & 1 & 1 & 1 & 0 & 1 & 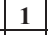 & 0 & 1 & 0 & 0 & 0 & 1 & 1 & 0 & 1 & 0 & 1 & 0 & 0 & 0 \\
\hline APP & $\mathbf{0}$ & 0 & 0 & 1 & 1 & 0 & 1 & 0 & 1 & 0 & 0 & 1 & 0 & 1 & 1 & 1 & 1 & 0 & 1 & 0 & 0 & 1 & 1 & 1 & 0 & 1 & 1 & 0 & 1 & 0 & 0 & 0 & 1 & 1 & 0 & 1 & 0 & 1 & 0 & 0 & 0 \\
\hline $\mathrm{BI}$ & 0 & 0 & 0 & 1 & 1 & 0 & 1 & 0 & 1 & 0 & 0 & 1 & 0 & 1 & 1 & 1 & 1 & 0 & 1 & 0 & 0 & 1 & 1 & 1 & 0 & 1 & 1 & 0 & 1 & 0 & 0 & 0 & 1 & 1 & 0 & 1 & 0 & 1 & $\mathbf{0}$ & 0 & 0 \\
\hline $\mathrm{ABH}$ & 0 & 0 & 0 & 1 & 1 & 0 & 1 & 0 & 1 & 0 & 0 & 1 & 0 & 1 & 1 & 1 & 1 & 0 & 1 & 0 & 0 & 1 & 1 & 1 & 0 & 1 & 1 & 0 & 1 & 0 & 0 & 0 & 1 & 1 & 0 & 1 & 0 & 1 & 0 & 0 & 0 \\
\hline $\mathrm{SA}$ & 0 & 0 & 1 & 0 & 0 & 0 & 0 & 0 & 0 & 0 & 1 & 0 & 1 & 0 & 0 & 0 & 0 & 0 & 0 & 0 & 1 & 0 & 0 & 0 & 0 & 0 & 0 & 1 & 0 & 1 & 1 & 1 & 0 & 0 & 1 & 0 & 1 & 0 & 0 & 1 & 0 \\
\hline
\end{tabular}

Bold characters were derived by comparative G-banding analysis.

mosome 6 and its L. lagothricha homolog appear to share an inversion with $A$. $b$. marginatus chromosome 5 not observed in C. apella or $A$. caraya (Fig. 2F). Finally, B. arachnoides chromosome 20 and its $C$. apella homolog probably share the acrocentric chromosome form, from which the submetacentric homologs of L. lagothricha and A. b. marginatus are derived by an inversion (Fig. 2G).

\section{Phylogenetic analysis}

In a first analysis, homologous chromosomes and chromosome segments were identified by multi-directional chromosome painting in seven Atelinae species (four Ateles species, $B$. arachnoides, $L$. lagothricha and $A$. caraya, the latter considered to have conserved the majority of Alouatta ancestral chromosome forms) and in the phylogenetic outgroup C. apella. This data was translated into numerical characters according to Table 2. The resulting binary data matrix with all species included in this study is given in Table 3. Maximum parsimony analysis resulted in only one most parsimonious tree (76 steps long, consistency index $=1.00$; retention index $=1.00$, homoplasy index $=0.00$ ): genus Ateles formed a monophyletic group with $A$. $b$. marginatus as the most basal clade, followed by $A$. $p$. paniscus and finally a clade formed by $A$. geoffroyi and $A$. $b$. hybridus. Phylogenetic relationships between genera Ateles, Brachyteles, Lagothrix and Alouatta could not be resolved. This tree is illustrated in Fig. 3A.

A second analysis, including both the chromosome painting and the six characters derived from G-banding data (Tables 1 and 2), resulted in a tree with 82 steps (Fig. 3B). Both the consistency and retention indexes were 1.00. Within genus Ateles, both trees as well as the bootstrap values at each node were identical. Considering all four Atelinae genera, however, Alouatta appears as the most basal clade, followed by Brachyteles and finally Ateles and Lagothrix.

\section{Discussion}

Most classifications of New World monkeys, based on morphological and molecular studies, agree with the monophyly of Atelinae, comprising Brachyteles, Lagothrix, Ateles and Alouat$t a$. There is, however no consensus yet concerning phylogenetic relationships between Atelinae genera, nor within genus Ateles. Here we report on a comparative molecular cytogenetic study including members from all four Atelinae genera that included four species from genus Ateles. For this purpose we analyzed $A$. $b$. marginatus, $A$. p. paniscus and $B$. arachnoides by multi-directional chromosome painting. In the attempt to reconstruct chromosomal phylogenies we further included previously published data on A. geoffroyi (Morescalchi et al., 1997), A. b. hybridus (Garcia et al., 2002), L. lagothricha (Stanyon et al., 2001) and $A$. caraya (de Oliveira et al., 2002) using $C$. apella (Garcia et al., 2000, de Oliveira et al., 2002) as an outgroup.

Evolutionary chromosome rearrangements can be considered to be "rare genomic changes" (see Rokas and Holland, 2000 , for review) with low levels of convergence. We attempted to reconstruct the succession of chromosomal rearrangements that led from the putative Platyrrhini ancestor $(2 n=54$ chromosomes, Neusser et al., 2001) to extant Atelinae species, in order to be able to distinguish between ancestral and derived chromosome forms. Shared derived traits would indicate a closer phylogenetic relationship between species as compared to species that do not share this synapomorphism. In a second approach, we subjected the chromosomal characters observed to a maximum parsimony analysis (PAUP).

All species included in our study shared derived chromosome forms $14 / 15 \mathrm{a}_{1}, 7 \mathrm{a} / 5 \mathrm{a} / 7 \mathrm{a}, 5 \mathrm{~b}, 4 \mathrm{a}, 4 \mathrm{~b} / 15 \mathrm{a}_{2}, 4 \mathrm{c}, 1 \mathrm{a}_{1}$ and $1 \mathrm{a}_{2}$, further supporting the proposed Atelinae ancestral karyotype with $2 \mathrm{n}=62$ chromosomes (de Oliveira et al., 2002) and hence Atelinae monophyly (Fig. 3A). 


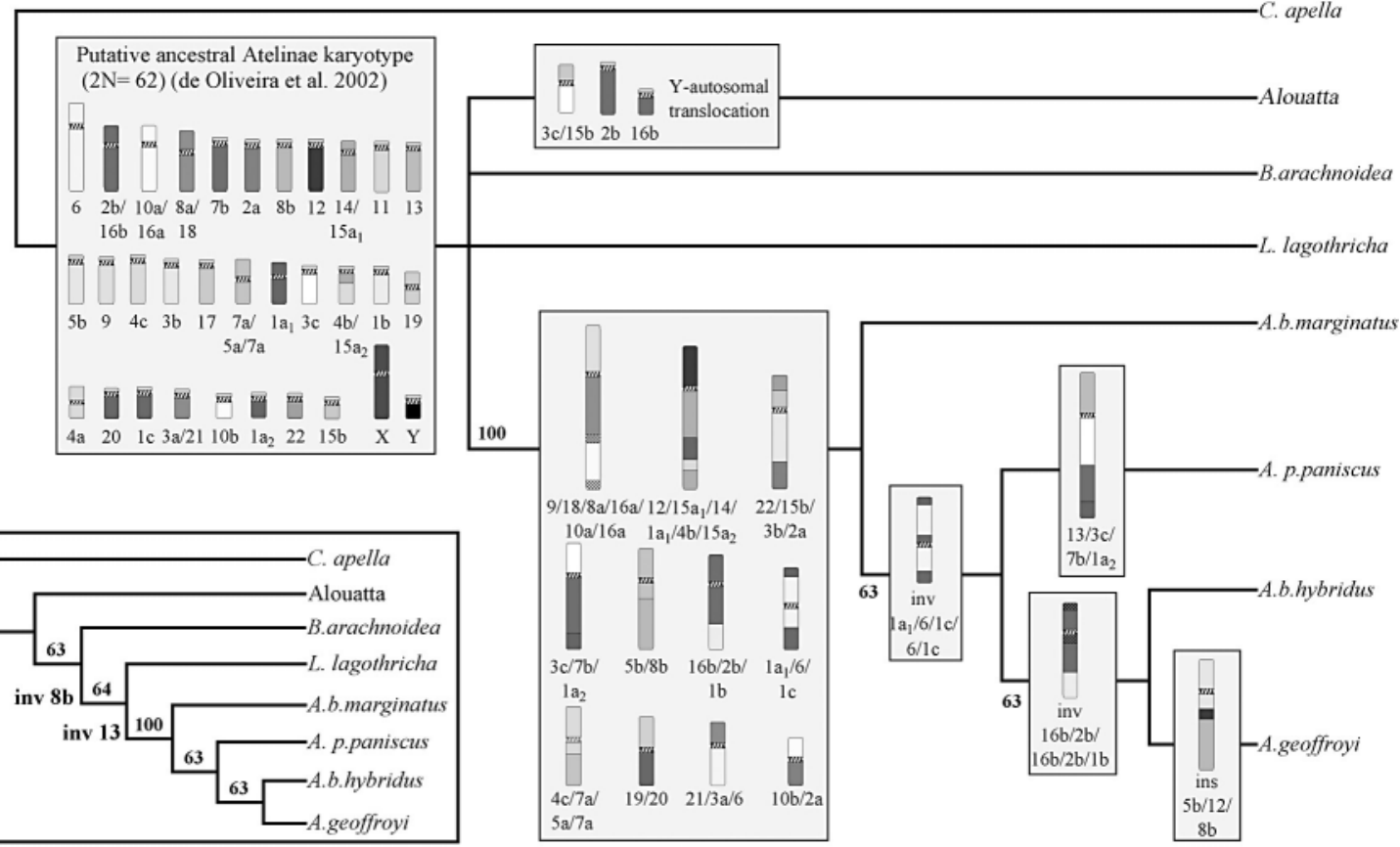

Fig. 3. Reconstruction of chromosomal phylogenies in Atelinae by a maximum parsimony approach (PAUP): (A) when employing only multi-directional chromosome painting data, a polytomy of all four genera was observed, while the branching sequence within genus Ateles was unequivocal. (B) This polytomy was resolved by the introduction of additional characters derived by comparative G-banding analysis, namely the inversions of the human chromosome $8 \mathrm{~b}$ and 13 homologues (see text for details). Bold numbers indicate bootstrap values. The chromosome nomenclature followed Neusser et al. (2001) and refers to homology with human chromosomes.

Concerning genus Ateles, 17 tandem or Robertsonian type fusions and three fissions are necessary to derive the putative ancestral Ateles karyotype conserved in A. b. marginatus $(2 \mathrm{n}=$ 34 ) from the ancestral Atelidae karyotype. Only $A$. b. marginatus chromosomes $11,12,14$ and 15 remain conserved, compared to the ancestral Atelidae karyotype. In the assumed last common ancestor of Ateles p. paniscus, A. geoffroyi and $A$. $b$. hybridus a pericentric inversion in chromosome form $1 \mathrm{a}_{1} / 6 / 1 \mathrm{c}$, leading to $1 \mathrm{a}_{1} / 6 / 1 \mathrm{c} / 6 / 1 \mathrm{c}$ (APP $7, \mathrm{ABH} 7$ and AGE 7) took place. A. geoffroyi and $A$. $b$. hybridus exclusively share the derived pericentric inversion $16 \mathrm{~b} / 2 \mathrm{~b} / 16 \mathrm{~b} / 2 \mathrm{~b} / 1 \mathrm{~b}$ on $\mathrm{ABH} 6$ and AGE 6 , whereas in $A$. $p$. paniscus a species-specific tandem fusion occurred, forming APP 4 (Fig. 2B) with the association of 13/ $3 \mathrm{c} / 7 \mathrm{~b} / 1 \mathrm{a}_{2}$. In $A$. geoffroyi a species specific insertion of human chromosome 12 homologous material occurred into AGE 5. This interpretation is in agreement with the hypothesis published by Medeiros et al. (1997), based on comparative G-banding analysis. However, it is discordant with the proposal of Collins and Dubach (2000) based on mitochondrial DNA variation, which placed $A$. p. paniscus as the most basal in comparison with $A$. b. marginatus and $A$. geoffroyi. Nevertheless, the cytogenetic evidence strongly argues for a reclassification of $A$. belzebuth hybridus, since its karyotype shares no synapomorphies exclusively with $A$. b. marginatus, but instead the derived inversion of chromosome 6 with $A$. geoffroyi.

When comparing putative ancestral karyotypes of Ateles and Alouatta, no shared derived chromosome forms between any of the extant species were observed, despite the occurrence of numerous chromosome rearrangements in both phylogenetic lines. The attempt to establish chromosomal phylogenies between the four genera employing chromosome painting characters alone, however, was hampered by the fact that both $B$. arachnoides and L. lagothricha conserved the ancestral Atelinae karyotype. This polytomy could be resolved by the introduction of additional chromosomal traits derived from comparative G-banding analysis. The derived inversion shared by $B$. arachnoides chromosome 6, L. lagothrich 7 and $A$. b. marginatus 5 may constitute a synapomorphism absent both in Alouatta and Cebus and argues for a close link between these three genera to the exclusion of genus Alouatta (Fig. 2F). Furthermore, considering that $B$. arachnoides chromosome 20 and its $C$. apella homolog share the ancestral acrocentric chromosome form, whereas the L. lagothricha and Ateles b. marginatus homologs are derived by a synapomorphic inversion (Fig. 2G), genera Ateles and Lagothrix would share a last common ancestor. Dutrillaux et al. (1986) introduced a similar hypothesis on the basis of comparative R-banding analysis; however, under the assumption that Lagothrix and Brachyteles would differ by three inversions and one translocation. The investigators further argued that Ateles and Lagothrix shared two characters not found in Brachyteles, whereas our results revealed only one derived chromosome form shared by Ateles and Lagothrix to the exclusion of Brachyteles and Cebus (Fig. 2E-G).

Alouatta as the sister group of the clade comprising Brachyteles, Lagothrix and Ateles agrees with previous views based on morphological (Rosenberger, 1992) and molecular data 
(Schneider et al., 1993, 1996; Meireles et al., 1999). The grouping of Ateles and Lagothrix, however, is controversial. Kay (1990) argued in favor, Ford (1986) presented a trichotomy of Brachyteles, Lagothrix and Ateles, while Rosenberger (1992) grouped Brachyteles and Ateles together, with Lagothrix as sister-group. Schneider et al. (1993, 1996) and Meireles et al. (1999) grouped Lagothrix and Brachyteles, with Ateles as a sister-group.

In conclusion, the cladistic reconstruction based on the identification of ancestral versus derived chromosome forms and the PAUP analysis resulted in the same most parsimonious phylogenetic tree: a polytomy of the four Atelinae genera when considering only chromosome painting data and a branching sequence $\{$ Alouatta $\{$ Brachyteles $\{$ Lagothrix and Ateles $\}\}$, when additionally introducing characters from intra-chromosomal rearrangements that were only detected by G-banding. These results on the one hand emphasize the importance of intra- chromosomal rearrangements as cladistic markers, which to date have not yet been exploited to their full extent. On the other hand, these observations pinpoint the limitation of the multi-directional painting approach presented here. Despite an increased subregional definition, compared to the use of human painting probes alone, some inversions could only be visualized by a varying centromeric index. In this respect, classical banding analysis remains an important comparative cytogenetics tool, in particular in combination with the secure establishment of gross chromosomal homologies by chromosome painting. Nevertheless, the delineation of evolutionary intra-chromosomal rearrangements with subregional probes such as BACs can be considered to be superior. A detailed molecular cytogenetic reinvestigation of the two key inversions that occurred during Atelinae karyotype evolution promises to substantiate the current interpretation of Atelinae chromosomal phylogeny.

\section{References}

Canavez FC, Moreira MA, Ladasky JJ, Pissinatti A, Parham P, Seuanez HN: Molecular phylogeny of new world primates (Platyrrhini) based on beta2 microglobulin DNA sequences. Mol Phylogenet Evol 12:74-82 (1999).

Collins AC, Dubach J: Phylogenetic relationships of spider monkeys (Ateles) based on mitochondrial DNA variation. Int J Primatol 21:381-420 (2000).

Consigliére S, Stanyon R, Koehler U, Agoramoorthy G, Wienberg J: Chromosome painting defines genomic rearrangements between red howler monkey subspecies. Chromosome Res 4:264-270 (1996).

Consigliére S, Stanyon R, Koehler U, Arnold N, Wienberg J: In situ hybridisation (FISH) maps chromsomal homologies between Alouatta belzebul (Platyrrhini, Cebidae) and other primates and reveals extensive interchromosomal rearrangements between howler monkey genomes. Am J Primato 46:119-133 (1998)

de Boer LEM, de Bruijn M: Chromosomal distinction between the red-faced and the black-faced black spider monkeys (Ateles paniscus paniscus and A. p. chamek). Zoo Biol 9:307-316 (1990).

de Oliveira EHC, Neusser M, Figueiredo WB, Nagamachi CY, Pieczarka JC, Sbalqueiro IJ, Wienberg J, Müller S: The phylogeny of howler monkeys (Alouatta, Platyrrhini): Reconstruction by multicolor cross-species chromosome painting. Chromosome Res 10:669-683 (2002).

Dutrillaux B, Coutourier J, Fosse AM: The use of highresolution banding in comparative cytogenetics: comparison between man and Lagothrix lagothricha (Cebidae). Cytogenet Cell Genet 27:45-51 (1980).

Dutrillaux B, Couturier J, Viegas-Péquignot E: Evolution chromosomique de Platyrhiniens. Mammalia 50:56-81 (1986).

Ford SM: Systematics of New World monkeys, in Swindler DR, Erwin J (eds): Comparative Primate Biology, Volume I: Systematics, Evolution and Anatomy, pp 73-135 (Alan R Liss, New York 1986).

Froehlich JW, Supriatna J, Froehlich PH: Morphometric analysis of Ateles: systematics and biogeographic implications. Am J Primatol 25:1-22 (1991).

Garcia F, Nogués C, Garcia M, Ponsà M, Ruiz-Herrera A, Egozcue J, Caldés MG: Chromosomal homologies between human and Cebus apella (Primates) revealed by ZOO-FISH. Mamm Genome 11:399401 (2000).
Garcia F, Ruiz-Herrera A, Egozcue J, Ponsa M, Garcia M: Chromosomal homologies between Cebus and Ateles (primates) based on ZOO-FISH and Gbanding comparisons. Am J Primatol 57:177-188 (2002).

Harada ML, Schneider H, Schneider MPC, Sampaio IM, Czelusniak J, Goodman M: DNA evidence on the phylogenetic systematics of New World monkeys: Support for the sister-grouping of Cebus and Saimiri from two unlinked nuclear genes. Mol Phyl Evol 4:331-349 (1995).

Kay RF: The phyletic relationships of extant and fossil Pithecinae (Platyrrhini). J Hum Evol 19:175-208 (1990).

Kellogg R, Goldman EA: Review on the spider monkeys. Proc US Mus Nat Hist 96:1-45 (1944).

Koiffmann CP: Variabilidade cromossomica na familia Cebidae (Platyrrhini, Primates). PhD thesis, Instituto de Biologia, USP, Sao Paulo, Brazil (1977).

Kunkel LM, Heltne PG, Borgaonkar DS: Chromosomal variation and zoogeography in Ateles. Int J Primatol 3:223-232 (1980).

Medeiros MA, Barros RM, Pieczarka JC, Nagamachi CY, Ponsa M, Garcia M, Garcia F, Egozcue J: Radiation and speciation of spider monkeys, genus Ateles, from the cytogenetic viewpoint. Am J Primatol 42:167-178 (1997).

Meireles CM, Czelusniak J, Schneider MPC, Muniz J, Brigido MC, Ferreira HS, Goodman M: Molecular phylogeny of Ateline New World monkeys (Platyrrhini, Atelinae) based on $\gamma$-globin pseudogene sequences: evidence that Brachyteles is the sister group of Lagothrix. Mol Phyl Evol 12:10-30 (1999).

Morescalchi MA, Schempp W, Wienberg J, Stanyon R: Chromosome painting in the New World monkey, Ateles geoffroyi, the black-handed spider monkey. Chromosome Res 5:527-536 (1997).

Müller S, Neusser M, Stanyon R, Wienberg J: Molecular cytogenetic characterization of EBV producing cell line B95-8 (Saguinus oedipus, Platyrrhini) by flow cytometry and multicolor cross species chromosome painting. Chromosome Res 9:689-693 (2001).

Neusser M, Stanyon R, Bigoni F, Wienberg J, Müller S: Molecular cytotaxonomy of Platyrrhini - comparative analysis of five species by multicolor reciprocal chromosome painting gives evidence for a classification of Callimico goeldii with Callitrichidae. Cytogenet Cell Genet 94:206-215 (2001).
Péquignot E, Koiffmann CP, Dutrillaux B: Chromosomal phylogeny of Lagothrix, Brachyteles and $\mathrm{Ca}$ cajao. Cytogenet Cell Genet 39:99-104 (1985).

Pieczarka JC, Nagamachi CY, Barros RMS: The karyotype of Ateles paniscus paniscus (Cebidae, Primates): $2 \mathrm{n}=32$. Braz J Genet 12:543-551 (1989).

Porter CA, Czelusniak J, Schneider H, Schneider MPC, Sampaio I, Goodman M: Sequences of the primate epsilon-globin gene: Implications for systematics of the marmosets and other New World primates. Gene 205:59-71 (1997a).

Porter CA, Page SL, Czelusniak J, Schneider H, Schneider MPC, Sampaio I, Goodman M: Phylogeny and evolution of selected primates as determined by sequences of the globin locus and $5^{\prime}$ flanking regions. Int $\mathrm{J}$ Primatol 18:261-295 (1997b).

Rokas A, Holland WH: Rare genomic changes as a tool for phylogenetics. Trends Ecol Evol 15:454-459 (2000).

Rosenberger AL: Evolution of New World primates, in Jones S, Martin R, Pilbeam D (eds): The Cambridge Encyclopedia of Human Evolution (Cambridge University Press, Cambridge UK 1992).

Schneider H, Schneider MPC, Sampaio MIC, Harada ML, Stanhope M, Goodman M: Molecular phylogeny of the New World monkeys (Platyrrhini, Primates). Mol Phylogenet Evol 2:225-242 (1993).

Schneider H, Sampaio I, Harada ML, Barroso CML, Schneider MPC, Czeluniak J, Goodman M: Molecular phylogeny of the New World monkeys (Platyrrhini, Primates) based on two unlinked nuclear genes: IRBP intron 1 and $\varepsilon$-globin sequences. Am J Phys Anthropol 100:153-179 (1996).

Stanyon R, Consigliére S, Bigoni F, Ferguson-Smith M, O'Brien PC, Wienberg J: Reciprocal chromosome painting between a New World primate, the woolly monkey, and humans. Chromosome Res 9:97-106 (2001).

Swofford D: PAUP*. Phylogenetic Analysis Using Parsimony (*and Other Methods). Version 4 (Sinauer Associates, Sunderland, MA 1998).

von Dornum M, Ruvolo M: Phylogenetic relationships of the New World monkeys (Primates, Platyrrhini) based on nuclear G6PD DNA sequences. Mol Phylogenet Evol 11:459-476 (1999). 